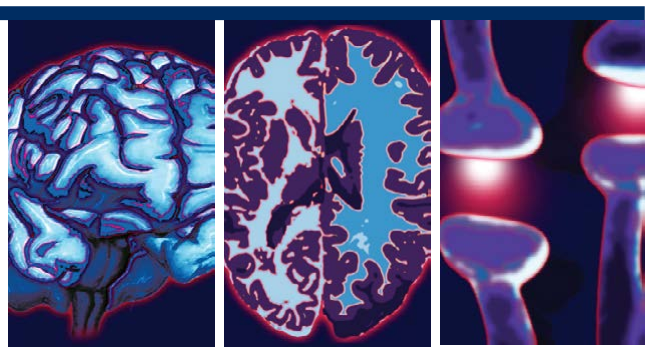

\title{
Methylphenidate-Elicited Distinct Neuropharmacological Activation Patterns Between Medication-Naïve Attention Deficit Hyperactivity Disorder Children With and Without Comorbid Autism Spectrum Disorder: A Functional Near- Infrared Spectroscopy Study
}

\begin{abstract}
Tatsuya Tokuda ${ }^{1,10, *}$, Takahiro Ikeda ${ }^{2,10, *}$, Yukifumi Monden ${ }^{1,2,3,4,10 \dagger}$, Sakae G Mizushima ${ }^{1,10}$, Takeshi Inoue ${ }^{6,10}$, Masako Nagashima ${ }^{2,10}$, Keiichi Shimamura ${ }^{6,10}$, Akari Arakawa ${ }^{6,10}$, Megumi Kobayashi ${ }^{7,10}$, Chie Kuroiwa ${ }^{6,10}$, Yuta Ujiie $^{8,10}$, Haruka Dan ${ }^{1}$, Yasushi Kyutoku' ${ }^{1}$,Takamichi Taniguchi ${ }^{4}$, Hideo Shimoizumi ${ }^{5}$, Takanori Yamagata ${ }^{2}$, Masami K Yamaguchi ${ }^{8,10}$, So Kanazawa ${ }^{9,10}$, Ryoichi Sakuta ${ }^{6,10}$, Ippeita Dan $^{1,10}$
\end{abstract}

\begin{abstract}
Objective:

We aimed to clarify the validity of fNIRS measurement for assessing the methylphenidate $(\mathrm{MPH})$ induced neuropharmacological effect in medication-naïve children with attention deficit hyperactivity disorder (ADHD) with or without comorbid autism spectrum disorder (ASD), thereby providing the first evidence for differing neurofunctional pathology between the two groups.
\end{abstract}

\section{Methods:}

We monitored the effects of a single acute clinical dose of MPH on the cortical hemodynamics of 32 medication-naïve $A D H D$ children ( $A D H D$ with $A S D n=11$ and $A D H D$ without $A S D n=21$ ) performing a go/no-go task before and $1.5 \mathrm{~h}$ after MPH or placebo administration, using a randomized, double-blind, placebo-controlled, crossover design. In addition, all subjects' symptoms were monitored using the Japanese version of the ADHD Rating Scale-IV (ADHDRS-IV-J) before and one month after MPH administration.

\footnotetext{
'Applied Cognitive Neuroscience Laboratory, Chuo University, 1-13-27 Kasuga, Bunkyo, Tokyo 112-8551, Japan

2Department of Pediatrics, Jichi Medical University, 3311-1 Yakushiji, Shimotsuke, Tochigi 329-0498, Japan

${ }^{3}$ Department of Pediatrics, International University of Health and Welfare, 537-3 Iguchi, Nasushiobara, Tochigi 329-2763, Japan ${ }^{4}$ International University of Health and Welfare, 2600-1 Kitakanemaru, Otawara, Tochigi, 324-8501, Japan

${ }^{5}$ Rehabilitation Center, International University of Health and Welfare, 2600-7 Kitakanemaru, Otawara, Tochigi 324-0011, Japan

${ }^{6}$ Child Development and Psychosomatic Medicine Center, Dokkyo Medical University Saitama Medical Center, 2-1-50 Minamikoshigaya, Koshigaya, Saitama 343-8555, Japan

${ }^{7}$ Department of Functioning Science, Institute for Developmental Research, Aichi Human Service Center, 713-8, Kamiya, Kasugai, Aichi 480-0392, Japan

${ }^{8}$ Department of Psychology, Chuo University, 742-1, Higashinakano, Hachioji, Tokyo 192-0393, Japan

'Department of Psychology, Japan Women's University, 1-1-1, Nishi-ikuta, Tama, Kawasaki, Kanagawa 214-8565, Japan ${ }^{10}$ RISTEX (Research Institute of Science and Technology for Society) Group

${ }^{\dagger}$ Author for correspondence: Yukifumi Monden, Department of Pediatrics, International University of Health and Welfare, 537-3 Iguchi, Nasushiobara, Tochigi 329-2763, Japan, Tel: +81 28558 7710; Fax: +81 28544 8329; email: mon4441977319@jichi.ac.jp

*These two authors contributed equally to this work.
} 


\section{Results:}

Medication-naïve ADHD children without ASD exhibited reduced right prefrontal activation. MPH medication elicited significant right prefrontal activation except in comparative conditions associated with the placebo effect. Medication-naïve ADHD children with ASD exhibited marginal activation before the first day of medication and reduced activation after $\mathrm{MPH}$ medication in the right prefrontal area. While the neuropharmacological effects of MPH were different, ADHD-RS-IV-J scores were significantly improved one month after MPH administration in ADHD groups both with and without ASD.

\section{Conclusion:}

Taken together, these results suggest that ADHD with ASD is characterized by a different underlying neurofunctional pathology for inhibition control than that without ASD. To our knowledge, this is the first comparative neuroimaging study between ADHD patients with and without comorbid ASD.

\section{Keywords}

Developmental disorders, Optical topography, Attention network, Response inhibition, Methylphenidate, Attention-deficit hyperactivity disorder, Autism spectrum disorder

\section{Introduction}

Attention deficit hyperactivity disorder (ADHD) is characterized by a clinical phenotype of hyperactivity, impulsivity and inattention [1], and is among the most common neuropsychiatric disorders in childhood, affecting from 3 to $7 \%$ of school-aged children [2,3]. The symptoms of ADHD in children typically developed during their preschool years [4]. Moreover, ADHD children tend to undergo social and emotional problems that cause academic difficulties and antisocial behaviors [5]. Therefore, early identification and treatment are important to improving the quality of life of ADHD patients [6].

The American Academy of Pediatrics (AAP) has recommended both medication and nonmedication (e.g. behavioral and/or community) treatments for ADHD children. For school-aged children, a considerable amount of evidence provides support for the recommendation of the administration of psychostimulants, such as methylphenidate (MPH), as the first-choice treatment $[7,8]$. $\mathrm{MPH}$ has been reported as the most effective treatment, improving attention and behavior as well as cognition and social function [9] in $70 \%$ of patients [10,11]. $\mathrm{MPH}$ inhibits the reuptake of catecholamines, especially dopamine, by blocking their transporters, and consequently acts as a dopamine inducer in the cerebral cortices and striatal regions [12]. The effect of MPH as a dopamine inducer makes it a reasonable treatment for $\mathrm{ADHD}$ from a genetic perspective as ADHD is thought to be associated with several dopamine-related genes, including the catechol-O-methyltransferase (COMT) gene [13], the dopamine receptor D4 (DRD4) gene and the dopamine active transporter 1 gene (DAT1, also known as SLC6A3) [14] at the prefrontal cortex in ADHD.

To diagnose ADHD and evaluate treatment, clinicians usually refer to the severity levels of symptoms listed on rating questionnaires for subjective measures of $\mathrm{ADHD}$ symptoms, including the ADHD Rating Scale and the Clinical Global Impression-Improvement of Illness scale [15]. However, the rating of symptoms is usually conducted by children's parents or teachers, and tends, unfortunately, to be subjective. Therefore, more objective approaches are urgently required [16]. In addition, more than four weeks of investigation is necessary to assess whether a patient is a responder or a non-responder to $\mathrm{MPH}$ [15]. Since MPH treatment has side effects such as appetite loss, tics, insomnia and stomachaches [17], discrimination between responders and non-responders should be done as quickly as possible. However, an objective biomarker for its pharmacological effect has yet to be established. Therefore, in order to ensure successful early diagnosis and treatment with medication of ADHD children, it is important to establish an objective biomarker to assess the effectiveness of $\mathrm{MPH}$ as early as possible after its initiation in medication-naïve ADHD children.

The use of noninvasive functional neuroimaging methods, such as functional magnetic resonance imaging (fMRI), single photon 

A Functional Near-Infrared Spectroscopy Study

emission computed tomography (SPECT), positron emission tomography (PET), and, lately, functional near-infrared spectroscopy (fNIRS), is one potential approach to detecting candidate biomarkers for ADHD. Among these neuroimaging modalities, fNIRS offers several advantages such as its usefulness in an ordinary examination room, accessibility and tolerance to body motion [18-28], and, thus, is suitable for the objective diagnosis of ADHD children and for assessing their treatment. Since the 2000s, fMRI research has examined pharmacological effects in ADHD from the perspective of brain function [29-31]. In general, these studies have revealed that the blood-oxygenation-leveldependent signal within the prefrontal cortex (PFC) is related to administration of ADHD therapeutic agents. However, because of the high elimination rate of fMRI measurement, amounting to the rejection of $50 \%$ of ADHD children due to excessive motion artifacts [32], the efficacy of fMRI may be limited for clinical applications.

However, research into the clinical application of fNIRS to monitor the effects of neurostimulants on cortical hemodynamic changes is currently being undertaken. Thus far, to our knowledge, there have been eleven reports on fNIRS-based exploration of neuropharmacological assessment of ADHD children since 2011 [33-44]. Ishii et al. performed a double-blind, placebocontrolled, crossover trial with a single dose of $\mathrm{MPH}$ and used ANIRS to measure hemodynamic responses during a stop-signal task [33]. They conducted a prospective 4- to 8-week open trial with continuous $\mathrm{MPH}$ administration, and found that ANIRS predicted behavioral performance changes in ADHD children after at least 4 weeks of MPH administration. Araki et al. evaluated the long-term effects of atomoxetine (ATX) and revealed improved hemodynamic response in the right dorsolateral PFC during a continuous-performance task [34]. Nakanishi et al. also reported the long-term effects of ATX in the left PFC during Stroop color-word task [35]. Matsuura et al. examined the effect of $\mathrm{MPH}$ administration on ADHD children without pervasive developmental disorder and reported changes of PFC activation during a spatial working-memory task [39]. Schecklmann et al. revealed normalization of brain activation in the temporal cortex under MPH medication during olfactory stimulation [41]. Sanefuji et al. reported the elevation of left ventrolateral PFC activation after $\mathrm{MPH}$ administration during a short-term memory task [42]. All of the studies described above included children with ADHD aged 6-16 years as subjects.

Meanwhile, in search of a stable biological marker, we have thus far explored the utility of the fNIRS monitoring method as a clinical tool for early diagnosis and treatment of ADHD children. Specifically, we applied an fNIRS-based clinically oriented evaluation method with young ADHD children, and accumulated evidence of neuro-functional modulation of the right inferior and middle frontal gyri (IFG/MFG), which is critical in inhibitory function, induced by $\mathrm{MPH}$ [40]. Subsequently, we examined the pharmacological effects of MPH [40] and ATX [38] in a randomized, double-blind, placebocontrolled, crossover study, revealing that both medications modulated hemodynamic responses in the right IFG/MFG during a go/no-go task while a placebo did not. Similar hemodynamic modulations after both medications were also observed when an oddball task was adopted $[36,37]$.

Furthermore, fNIRS has successfully allowed visualization of the differential neural substrate between ADHD and healthy control children in inhibitory $[38,40,43]$ and attentional $[34,35]$ tasks with group analyses. While a go/no-go task recruits the right IFG/MFG in control children, this activation is absent in ADHD children. These findings led us to postulate that the right IFG/MFG activations as observed using fNIRS, especially for go/no-go tasks, would serve as an objective neuro-functional biomarker to diagnose school-aged ADHD children, possibly even at the individual level.

Subsequently, we succeeded in individually differentiating ADHD children from typicallydeveloping control children using multichannel fNIRS with an area under a receiver operating characteristic (ROC) curve value of $90 \%$ and with sensitivity and specificity over $70 \%$. This is promising efficacy for individual diagnosis of ADHD children [44]. These findings led us to conclude that the activation in the IFG/MFG could serve as an objective neuro-functional biomarker associated with a specific core neuropathological mechanism for inhibitory control in ADHD children. However, in order to open up our fNIRS measurement tool as the template method for the objective clinical diagnosis and treatment of ADHD children, we must consider an important issue: the complication of autism spectrum disorder (ASD). 
In our 2012 study (see Figure 4 on clinical neurophysiology in [40]), we reported preliminary findings for unique, MPH-elicited neuropharmacological activation between ADHD with and without comorbid ASD; although the association between a go/no-go paradigm and right IFG/MFG activation as a biological marker is expected to be stable in ADHD without ASD, this may not be the case for ADHD with ASD.

Until 2012, the DSM-IV ADHD diagnostic criteria had specified that an ASD diagnosis was an exclusion criterion for ADHD, which resulted in limited research of the common clinical comorbidity [14]. However, in 2013, the DSM-5, in its revised criteria, recognized the frequency of the occurrence of ADHD with ASD and allowed, for the first time, a comorbid diagnosis of ADHD with ASD [1,14]. Indeed, an increasing number of clinical [45] and psychological [46] studies on the co-occurrence of ADHD and ASD have been published since 2013. Concerning pharmacological studies, the response rate for $\mathrm{MPH}$ has been reported as lower in ADHD with ASD than in ADHD alone [47]. In addition, while MPH may worsen irritability in ADHD with ASD, it appears to improve it in ADHD without ASD [47].

Although ADHD with and without ASD may have important differences in core neurofunctional pathology, to date neurophysiological and neuroimaging evidence has only been presented in two pioneering studies that used electroencephalogram (EEG) [48] and fMRI [49]. The EEG study on eventrelated potentials (ERPs) during a continuous performance task demonstrated different ERP patterns between ADHD with ASD, ADHD without ASD and ASD without ADHD in 8to 13-year-olds [48]. On the other hand, the
fMRI study demonstrated that ADHD patients with ASD aged 11-17 years exhibited a greater association of a temporal discounting task with several brain regions, including the PFC, than did control and non-comorbid groups [49]. Therefore, a better understanding of the neuropharmacological endophenotype of the comorbidity of ADHD with ASD is important because it could provide clues for new treatment options as well as objective evidence for the existence of a distinct phenotype [46]. However, to our knowledge, no neuroimaging study has explored the differences in neuropharmacological effects between ADHD with ASD and ADHD without ASD. When this history and these results are taken together, it is clear that the time has come to explore the neuropathology of ADHD with and without comorbid ASD using fNIRS measurement. Hence, in the current study, we extended our previous fNIRS studies to include the assessment of medication-naïve ADHD children with and without comorbid ASD in order to examine the neuropharmacological effect of MPH on the cortical hemodynamics of ADHD during a go/no-go task. ADHD children received either $\mathrm{MPH}$ or a placebo according to a randomized, double-blind, placebo-controlled, crossover design. We hypothesized that $\mathrm{MPH}$ would modulate hemodynamic responses differently in ADHD children with ASD and ADHD children without ASD.

\section{Materials and Methods \\ - Participants and ethics}

Thirty-two clinically referred medication-naïve, right-handed Japanese children, diagnosed as ADHD based on the DSM- 5 by trained pediatric neurologists and requiring the administration of $\mathrm{MPH}$, were randomly recruited at Jichi Medical University and the Rehabilitation Center of the

Table 1: Patient characteristics ( $n=32)$.

\begin{tabular}{|c|c|c|c|c|}
\hline & & $\begin{array}{l}\text { Gender } \\
\text { male:female }\end{array}$ & $\begin{array}{l}\text { Age } \\
\text { (years) }\end{array}$ & WISC-III or IV Full IQ \\
\hline \multirow[t]{2}{*}{ ADHD with ASD $(n=11)$} & mean & 11:0 & 8.2 & 103.2 \\
\hline & SD & - & 2.1 & 14.5 \\
\hline \multirow[t]{2}{*}{ ADHD without ASD $(n=21)$} & mean & $17: 4$ & 7.8 & 92.8 \\
\hline & SD & - & 1.7 & 12.9 \\
\hline \multirow[t]{4}{*}{ ADHD with ASD vs ADHD without ASD } & $\chi^{2}$ & 2.395 & - & - \\
\hline & $t$ & - & 0.614 & 2.076 \\
\hline & $p$ & 0.122 & 0.544 & 0.046 \\
\hline & sig & ns & ns & * \\
\hline
\end{tabular}

Abbreviations: WISC-III or IV, Wechsler Intelligence Scale of Children third or fourth edition; IQ, intelligence quotient; SD, standard deviation; $\chi 2$, Chisquared; $t, t$ value; $p, p$ value; sig, statistical significance. Statistical significances are presented as follows: ${ }^{*} p<0.05$; and ns, not significant. 
International University of Health and Welfare. It was also determined whether participants met the DSM-5 criteria for ASD. Consequently, subjects included eleven ADHD children with ASD (mean age $=8.2$, SD 2.1, range 7 to 14 years; 11 male) and twenty-one ADHD without ASD (mean age $=7.8$, SD 1.7, range 6 to 13 years; 17 male and 4 female) (Table 1). Their full scale IQ scores were assessed by the Wechsler Intelligence Scale of Children Third (WISC-III) or Fourth (WISC-IV) and the FSIQ was above 70 children were included in this study. IQ scores of ADHD subjects with ASD (mean 103.2, SD 14.5, range 76 to 129$)$ were significantly higher $(t(30)=2.076$, $\mathrm{p}=0.046)$ than those of ADHD subjects without ASD (mean 92.8, SD 12.9, range 73 to 120 ).

In this study, we selected medication-naïve ADHD children. Although our previous studies included both medicated and medication-naïve patients, according to animal [50] and human anatomical studies [51-53], brain function and structure can change with long-term $\mathrm{MPH}$ administration.

All participants and their parents gave oral consent to participation in the study. Written consent was obtained from the guardians of all subjects according to the latest version of the Declaration of Helsinki. The study was approved by the Ethics Committees of Jichi Medical University Hospital and the International University of Health and Welfare.

\section{- Experimental design}

The effects of $\mathrm{MPH}$ were evaluated in a randomized, double-blind, placebo-controlled, crossover design, using $\mathrm{MPH}(18 \mathrm{mg})$. The participants visited either of the two hospitals twice, and were medicated with $\mathrm{MPH}$ during their first visit and a placebo during their second visit, or vice versa. Their order was allocated in a pseudo-randomized order to avoid order effects, and counter-balanced across all patients. In order to avoid patients' own biased expectations of the results, we adopted a double-blind design in which neither researchers nor patients knew whether a placebo or MPH was administered. Cortical activation was measured with fNIRS during a go/no-go task. Experimental procedure was identical to Monden (2012).

In addition, to evaluate the pharmacological effect, a Japanese version of the ADHD Rating Scale-IV(ADHD-RS-IV-J) [54] was given before starting oral administration and 1 month after the current study. ADHD-RS-IV-J is an 18-item scale with each item corresponding to one of the 18 symptoms reflecting the DSM-IV diagnostic criteria for ADHD (American Psychiatric Association 1994; [55]). The questionnaire was responded to by each patient's parents.

\section{- Experimental stimulus}

We adopted the go/no-go task in a block design [25,56-59]. We presented four types of animal pictures on a computer screen using E-prime 2.0 (Psychology Software Tools). A go/no-go task consisted of six 2-block sets. Each set contained a go (baseline) block, where participants were randomly presented with two types of animal pictures and asked to respond to both pictures, and a go/no-go (target) block, where participants were randomly presented with a no-go picture $50 \%$ of the time and asked to respond to all the pictures except the no-go picture. Baseline and target blocks were alternated. Each block lasted for $24 \mathrm{~s}$ and was preceded by instructions for $3 \mathrm{~s}$ for a total session time of $6 \mathrm{~min}$. For the go block, participants were instructed, "when you see each picture, you should press the space key as quickly as you can". For the go/no-go block, subjects were instructed, "when you see the no-go picture, you should not press the space key". Pictures were presented sequentially for $800 \mathrm{~ms}$ with an inter-stimulus interval of $200 \mathrm{~ms}$ during go and go/no-go blocks.

\section{- fNIRS measurement}

We used the multichannel fNIRS system ETG4000 (Hitachi Corporation, Tokyo, Japan), using two wavelengths of near-infrared light $(695$ and $830 \mathrm{~nm})$. We analyzed the optical data based on the modified Beer-Lambert Law [60] as previously described [61]. Signals reflecting the oxygenated hemoglobin (oxy- $\mathrm{Hb}$ ), deoxygenated hemoglobin (deoxy-Hb), and total hemoglobin (total-Hb) changes were calculated in units of millimolar-millimeter $(\mathrm{mM} \cdot \mathrm{mm})$ [61,62]. The sampling rate was set at $10 \mathrm{~Hz}$. We analyzed oxy$\mathrm{Hb}$ signal as in previously described $[40,44]$.

\section{- fNIRS probe placement}

We set the fNIRS probes to cover IFG and MFG activation foci in reference to our previous studies $[38,40,43,44]$. We used 2 sets of $3 \times 5$ multichannel probe holders, consisting of 8 illuminating and 7 detecting probes arranged alternately at an interprobe distance of $3 \mathrm{~cm}$, resulting in 22 channels $(\mathrm{CH})$ per set. The midpoint of a pair of illuminating and detecting probes was defined as a channel location. The bilateral probe holders were attached 
a
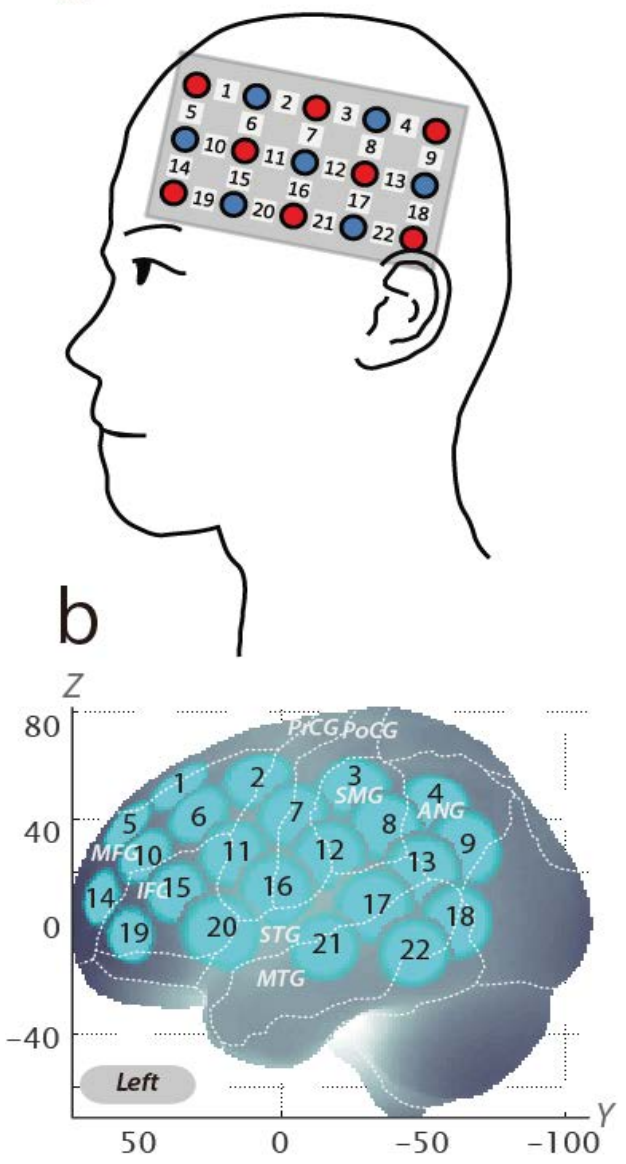
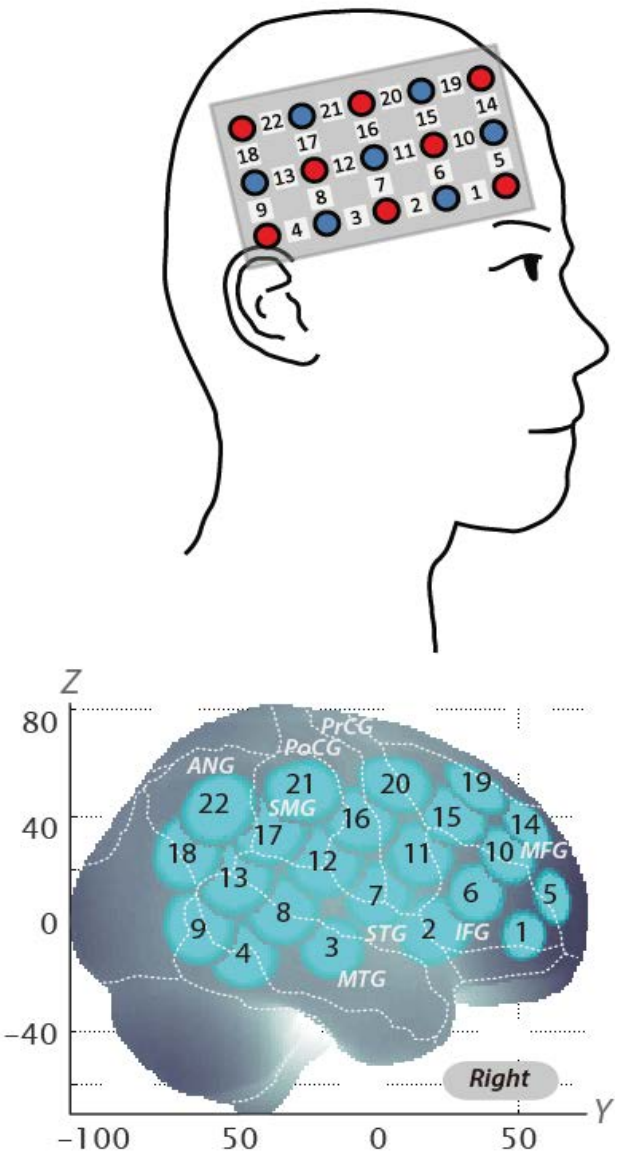

Figure 1: Spatial profiles of fNIRS channels. (a) Both side views of probe arrangements. fNIRS channel orientation is also illustrated. Detectors are shown as blue circles, illuminators as red circles, and channels as white squares. Corresponding channel numbers are indicated in black. (b) Channel locations on the brain. Right-side and left-side views are illustrated. Statistically estimated fNIRS channel locations (centers of blue circles) for ADHD with ASD and without ASD, and their spatial variability (SDs, radii of the blue circles) associated with the estimation are exhibited in MNI space.

\section{Table 2: Spacial profiles of the channel screened for involvement with go/no-go tasks.}

\begin{tabular}{|l|l|l|l|l|l|l|}
\hline \multicolumn{2}{|c|}{ MNI coordinates } & \multicolumn{4}{|c|}{} \\
\hline & $\mathbf{x}, \mathbf{y}, \mathbf{z}$ (SD) & Macroanatomy & prob & Broadmann area & prob \\
\hline CH10 & $48,46,26(13)$ & R middle frontal gyrus & 0.63 & 45 & pars triangularis Broca's area & 0.57 \\
\hline & & R inferior frontal gyrus & 0.38 & 46 & Dorsolateral prefrontal cortex & 0.44 \\
\hline Abbreviations: prob, probability; SD, standard deviation; R, right. & & & & \\
\hline
\end{tabular}

in the following manner: (1) their upper anterior corners, where the left and right probe holders were connected by a belt, were symmetrically placed across the sagittal midline; (2) the lower anterior corners of the probe holder were placed over the supraorbital prominence; (3) the lower edges of the probe holders were attached to the upper part of the auricles (Figure 1).

For spatial profiling of fNIRS data, we adopted the probabilistic registration method [63-65] to register fNIRS data to Montreal Neurological Institute (MNI) standard brain space. Specifically, the positions for channels and reference points, which included the $\mathrm{Nz}$ (nasion), $\mathrm{Cz}$ (midline central) and left and right preauricular points, were measured using a threedimensional digitizer in real-world (RW) space. We affine-transformed each RW reference point to the corresponding MRI-database reference point and then replaced them to MNI space. Adopting the same transformation parameters enabled us to obtain the MNI coordinate values for the fNIRS channels in order to obtain the most likely estimate of the location of given channels for the group of participants, and the 
Methylphenidate-Elicited Distinct Neuropharmacological Activation Patterns Between Medication-Naïve Attention Deficit Hyperactivity Disorder Children With and Without Comorbid Autism Spectrum Disorder: A Functional Near-Infrared Spectroscopy Study

spatial variability associated with the estimation (Table 2). Finally, the estimated locations were anatomically labeled using a MATLAB ${ }^{\circledast}$ function that reads anatomical labeling information coded in a microanatomical brain atlas, LBPA40 [66] and Brodmann's atlas [67].

\section{- Analysis of fNIRS data}

We preprocessed individual timeline data for the oxy-Hb signals of each channel with a first-degree polynominal fitting and high-pass filter using cutoff frequencies of $0.01 \mathrm{~Hz}$ to remove baseline drift, and a $0.8 \mathrm{~Hz}$ low-pass filter to remove heartbeat pulsations. From the preprocessed time series data, we computed channel-wise and participant-wise contrasts by calculating the inter-trial mean of differences between the oxy-Hb signals for target ( $4 \mathrm{~s}$ to end of task after go/no-go block onset) and baseline (14 to $24 \mathrm{~s}$ after go block onset) periods.

For the 6 go/no-go block sets, we removed the blocks with sudden, obvious, discontinuous noise based on independent visual examination by two raters. Cohen's Kappa coefficient for inter-rater consistency was 1.00. Furthermore, we excluded the data of participants for whom more than 3 blocks were removed.

\section{- Statistical analysis}

In order to explore the effect of $\mathrm{MPH}$ based on ADHD-RS-IV-J scores, we compared the scores (inattention, hyperactivity/impulsivity and total) for before and one month after starting oral administration of MPH. We performed paired t-tests (two tails) with an alpha level set at 0.05 .

We performed statistical analyses in a channelwise manner on oxy-Hb signals. For both groups, the following contrasts were generated: (1) firstday pre-medication contrast: target vs. baseline contrast (either PLA or MPH administration) for the first day exclusively; (2) post-medication contrasts (specifically, post-placebo and post$\mathrm{MPH}$ contrasts): target vs. baseline contrast for the post-PLA and post-MPH conditions; (3) intramedication contrasts: difference between post- and pre-medication contrasts for each medication (i.e., intra-PLA and intra-MPH contrasts); and (4) intermedication contrast: difference between intra$\mathrm{MPH}$ and intra-placebo contrasts.

We previously reported that right IFG/MFG activation in ADHD children was acutely normalized after administration of $\mathrm{MPH}$ $[36,40,43]$. Thus, we set the right $\mathrm{CH} 10$, located at the right IFG/MFG, as a region-of-interest (ROI) for the rest of the study. For the ROI, we performed paired t-tests (two tails) on target vs. baseline contrasts with an alpha level set at 0.05 .

To screen the neuropharmacological effects of $\mathrm{MPH}$, we performed comparisons between ADHD with ASD and ADHD without ASD for

\begin{tabular}{|c|c|c|c|c|c|c|c|}
\hline & \multicolumn{2}{|c|}{ Before starting medication } & \multicolumn{2}{|c|}{1 month after starting oral medication } & \multirow[b]{2}{*}{$t$} & \multirow[b]{2}{*}{$p$} & \multirow[b]{2}{*}{ sig } \\
\hline & Mean & SD & Mean & SD & & & \\
\hline \multicolumn{8}{|l|}{ ADHD with ASD $(n=11)$} \\
\hline Inattention score & 17.9 & 5.0 & 13.2 & 6.2 & 3.85 & 0.003 & ** \\
\hline Hyperactivity/Impulsivity score & 17.6 & 5.5 & 10.8 & 5.2 & 5.11 & 0.000 & **** \\
\hline Total score & 35.5 & 9.6 & 24.0 & 10.8 & 4.89 & 0.001 & **** \\
\hline \multicolumn{8}{|l|}{ ADHD without ASD ( $n=21)$} \\
\hline Inattention score & 17.9 & 5.1 & 11.2 & 4.0 & 4.87 & 0.000 & **** \\
\hline Hyperactivity/Impulsivity score & 12.6 & 5.8 & 8.8 & 5.0 & 3.44 & 0.003 & ** \\
\hline Total score & 30.5 & 9.2 & 20.0 & 8.2 & 4.72 & 0.000 & $* * *$ \\
\hline
\end{tabular}

Table 4: Go/no-go task functional data for both groups.

\begin{tabular}{|c|c|c|c|c|c|c|c|c|c|c|c|}
\hline & \multirow[t]{2}{*}{ Contrast } & \multicolumn{5}{|c|}{ ADHD with ASD $(n=11)$} & \multicolumn{5}{|c|}{ ADHD without ASD $(n=21)$} \\
\hline & & Mean & SD & $t$ & $p$ & sig & Mean & SD & $t$ & $p$ & sig \\
\hline \multirow{6}{*}{$\begin{array}{l}\Delta \text { Oxy-Hb } \\
(\mathbf{m M} \cdot \mathbf{m m})\end{array}$} & first-day pre-medication & 0.041 & 0.067 & 2.016 & 0.071 & t & 0.024 & 0.068 & 1.605 & 0.124 & ns \\
\hline & post-PLA & 0.073 & 0.114 & 2.128 & 0.059 & $\dagger$ & 0.057 & 0.085 & 3.060 & 0.006 & ** \\
\hline & post-MPH & -0.010 & 0.068 & -1.037 & 0.324 & ns & 0.060 & 0.117 & 3.991 & 0.001 & **** \\
\hline & intra-MPH & -0.047 & 0.107 & -1.446 & 0.179 & ns & 0.035 & 0.092 & 1.736 & 0.098 & $t$ \\
\hline & intra-PLA & 0.033 & 0.115 & 0.941 & 0.369 & ns & 0.008 & 0.120 & 0.296 & 0.770 & ns \\
\hline & inter-medication & -0.079 & 0.150 & -1.754 & 0.110 & ns & 0.027 & 0.178 & 0.694 & 0.495 & ns \\
\hline
\end{tabular}

Statistical significance are presented as follows: ${ }^{\dagger} p<0.1 ;{ }^{*} p<0.05 ;{ }^{* *} p<0.01 ;{ }^{* * *} p<0.001$; ns, not significant. Abbreviations: SD, Standard deviation; $t, t$ value; $p, p$ value; sig, statistical significance. 


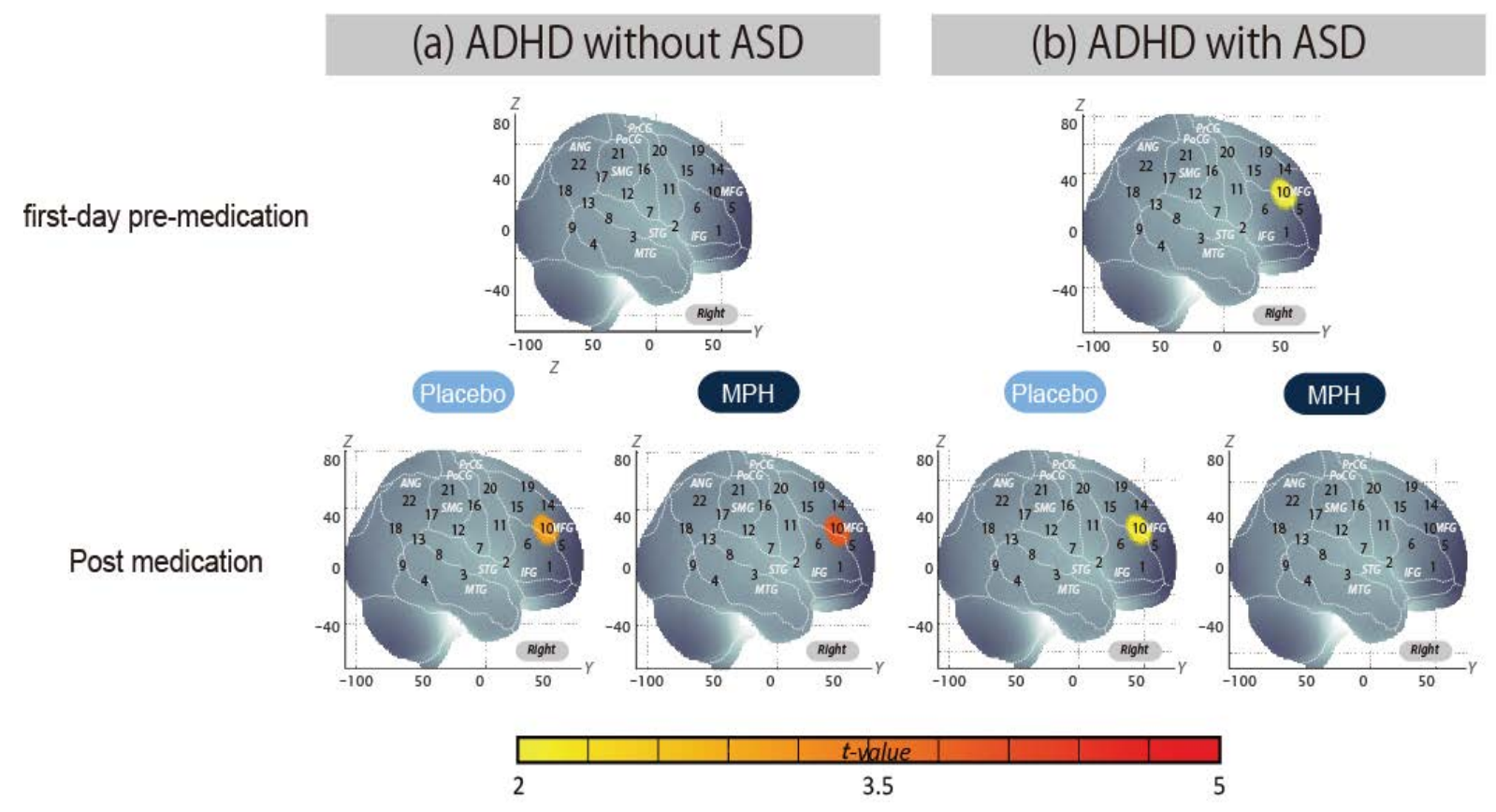

Figure 2: Cortical activation patterns of both participant groups: (a) ADHD without ASD and (b) ADHD with ASD. t-maps of oxy-Hb signals are displayed, with marginally significant and significant t-values (paired t-test) shown according to the color bar. All coordinates are in MNI space.

the following three contrasts for the right $\mathrm{CH} 10$ : (1) post-MPH, (2) intra-MPH, and (3) intermedication. We performed two sample t-tests (two-tails) on these contrasts with an alpha level set at 0.05 .

\section{Results}

\section{- Questionnaire results}

The results for the ADHD-RS-IV-J are summarized in Table 3. The ADHD-RS-IV-J scores 1 month after starting oral $\mathrm{MPH}$ were significantly lower than those before starting medication for ADHD both with and without ASD.

fNIRS examination of go/no-go task and MPH effects in ADHD without ASD

We examined oxy-Hb signal changes in the right $\mathrm{CH} 10$ for ADHD without ASD in the following contrasts: (1) first-day pre-medication, (2) postmedication, (3) intra-medication, and (4) intermedication. We found no significant activation in the right $\mathrm{CH} 10$ during the go/no-go task period of the first-day pre-medicated (paired t-test, $\mathrm{p}>0.05$, Cohen's $\mathrm{d}=0.35$ ), but significant activation in the post-MPH conditions (paired t-test, $\mathrm{p}<0.05$, Cohen's $\mathrm{d}=0.87$, Table 4, Figure 2).

Effects of medications were examined between pre-MPH/PLA and post-MPH/PLA. We observed no significant change in oxy-Hb signal for pre-MPH/PLA and post-MPH/PLA (paired t-test, $\mathrm{p}>0.05$, intra-PLA: Cohen's $\mathrm{d}=0.06$, intraMPH: Cohen's d=0.37, Table 4).

Finally, we tested whether there was an MPHinduced, but not PLA-induced activation. In the inter-medication contrast, the right $\mathrm{CH} 10$ was found to have no significant difference (paired t-test, $\mathrm{p}>0.05$, Cohen's d=0.15, Table 4).

fNIRS examination of go/no-go task and MPH effects in ADHD with ASD

We examined oxy-Hb signal changes in the right $\mathrm{CH} 10$ for ADHD with ASD in the following contrasts: (1) first-day pre-medication, (2) postmedication, (3) intra-medication, and (4) intermedication.

We found marginally significant activation in the right $\mathrm{CH} 10$ during the go/no-go task period of the first-day pre-medicated and post-PLA contrast, but a moderate effect size was obtained (paired t-test, $\mathrm{p}>0.05$, first-day pre-medication: Cohen's $\mathrm{d}=0.60$, post-PLA: Cohen's $\mathrm{d}=0.51$, Table 4, Figure 2).

Effects of medications were examined between pre-MPH/PLA and post-MPH/PLA. We observed no significant change in oxy-Hb signal for pre-MPH/PLA and post-MPH/PLA (paired t-test, $\mathrm{p}>0.05$, intra-PLA: Cohen's $\mathrm{d}=0.28$, intra$\mathrm{MPH}$ : Cohen's $\mathrm{d}=-0.43$, Table 4). 
Methylphenidate-Elicited Distinct Neuropharmacological Activation Patterns Between Medication-Naïve Attention Deficit Hyperactivity Disorder Children With and Without Comorbid Autism Spectrum Disorder: A Functional Near-Infrared Spectroscopy Study

Table 5: Comparison of neuropharmacological effect of MPH between ADHD with ASD and without ASD.

\begin{tabular}{|c|c|c|c|c|c|c|c|c|}
\hline & \multirow[t]{2}{*}{ Contrast } & \multicolumn{2}{|c|}{$\begin{array}{l}\text { ADHD with ASD } \\
(n=11)\end{array}$} & \multicolumn{2}{|c|}{$\begin{array}{l}\text { ADHD without ASD } \\
(n=21)\end{array}$} & \multicolumn{3}{|c|}{$\begin{array}{l}\text { ADHD with ASD vs ADHD without } \\
\text { ASD }\end{array}$} \\
\hline & & Mean & SD & Mean & SD & $t$ & $p$ & sig \\
\hline \multirow{3}{*}{$\Delta$ Oxy-Hb (mM $\cdot \mathbf{m m})$} & post-MPH & -0.010 & 0.068 & 0.060 & 0.117 & -3.030 & 0.005 & ** \\
\hline & intra-MPH & -0.047 & 0.107 & 0.035 & 0.092 & -2.255 & 0.032 & ** \\
\hline & inter-medication & -0.079 & 0.150 & 0.027 & 0.178 & -1.689 & 0.102 & ns \\
\hline
\end{tabular}

Finally, we tested whether there was an MPHinduced, but not PLA-induced activation. In the inter-medication contrast, the right $\mathrm{CH} 10$ was found to have insignificant difference (paired t-test, $\mathrm{p}>0.05$, Cohen's $\mathrm{d}=0.15$, Table 4).

\section{- Neuropharmacological effect of MPH comparison between ADHD with ASD and without ASD}

Comparison between oxy- $\mathrm{Hb}$ signals of the ADHD patients with ASD and those without ASD in post-MPH and intra-MPH conditions revealed significant activation of oxy- $\mathrm{Hb}$ signal in the right $\mathrm{CH} 10$ in the patients without ASD (independent two-sample t-test, $\mathrm{p}<0.05$, post$\mathrm{MPH}$ : Cohen's $\mathrm{d}=1.16$, intra-MPH: Cohen's $\mathrm{d}=0.86$, Table 5). In the inter-medication contrast, there was no statistical difference between groups, but a moderate effect size was obtained (independent two-sample t-test, p $>0.05$, Cohen's d=0.64, Table 5).

\section{Discussion}

\section{- Overview}

The current study aimed to explore the neural substrate of MPH effects on inhibition control in medication-naïve ADHD (with ASD $(n=11)$ and without ASD $(\mathrm{n}=21)$ ) school-aged children using fNIRS. We examined cortical activation in the ROI (right IFG/MFG), where we have consistently found significant activation during go/no-go task periods in control subjects $[40,44]$. In the ADHD without ASD group, $\mathrm{MPH}$ medication led to increased right IFG/MFG activation. Conversely, in the ADHD with ASD group, MPH medication resulted in decreased right IFG/MFG activation. Finally, in both groups, there was placebo-induced activation in the IFG/MFG, and thus activation in the intermedication (intra-MPH vs intra-PLA) contrast did not reach a significant level.

\section{- Placebo effect in medication-naïve ADHD}

We examined the placebo effect for several contrasts. At the right $\mathrm{CH} 10$, located in the
IFG/MFG, oxy-Hb signal during the go/no-go task period was significantly higher than that of the baseline period for ADHD without ASD subjects, and marginally significant in ADHD with ASD in post-PLA conditions. In addition, due to the placebo effect, $\mathrm{MPH}$ medication effects on ADHD without ASD exemplified in the inter-medication contrast failed to reach significance. These results suggest that regardless of $\mathrm{MPH}$-induced activation, a non-negligible placebo effect was present, inducing activation in medication-naïve ADHD children both with and without ASD.

The placebo effect was not observed in our past study [40], which employed the same protocol, including the go/no-go paradigm, double-blind, placebo-controlled and crossover designs for MPH effect. However, participants in the present study were all medication-naïve, while nine out of sixteen were not naïve in the previous study.

In general, more than $50 \%$ of clinical trial studies for new psychiatric medications have demonstrated statistical superiority for a placebo over the real medication $[68,69]$. It has been suggested that placebo response has increased because of the heightened expectations of patients and researchers for medication effects [70,71]. Therefore, the placebo-induced neuropharmacological effect found in the present study likely reflects the medicationnaïve $\mathrm{ADHD}$ patient's high expectations of $\mathrm{MPH}$. Although investigation into the factors that cause placebo responses is beyond the scope of the current research, we suggest that when exploring neuropharmacological effects using fNIRS, double-blind, placebo-controlled studies should be adopted to account for the impact of the placebo effect.

\section{n fNIRS examination of go/no-go task and MPH effects in ADHD without ASD}

We found no significant activation in the right IFG/MFG (CH10) during the go/no-go task period in the pre-medicated medication-naïve ADHD participants without ASD, but observed 
significant activation in the post-medicated conditions (Table 4). These results suggest that the right prefrontal function associated with go/no-go task performance was impaired in medicationnaïve $\mathrm{ADHD}$ children without $\mathrm{ASD}$.

The right prefrontal dysfunction and $\mathrm{MPH}-$ elicited recovery observed using ANIRS is consistent with former studies, with the exception of the placebo-related conditions [43]. Furthermore, these condition- and subject-dependent activation patterns are consistent with the results of previous fMRI studies on ADHD children in general, as well as those on ADHD children without ASD [58,72-74] (also see meta analyses by [75-77]). Taken together, this suggests that dysfunction of the right IFG/MFG during the initial go/no-go task and the subsequent MPH-induced recovery of right IFG/ MFG activation is robustly applicable to medicationnaïve $\mathrm{ADHD}$ children without ASD.

\section{- fNIRS examination of go/no-go task and MPH effects in ADHD with ASD}

To our surprise, MPH induced opposing neuropharmacological effects in ADHD with and in that without ASD. Specifically, we found marginal activation in the right IFG/MFG during the go/no-go task period in the pre-medicated medication-naïve ADHD subjects with ASD, but we found reduced activation in the postmedicated conditions (Table 4). Furthermore, comparison between MPH-induced postbaseline ADHD without ASD and ADHD with ASD revealed significantly different oxy$\mathrm{Hb}$ changes in the right IFG/MFG (CH10). Comparison between intra-MPH in ADHD without ASD and intra- MPH in ADHD with ASD also revealed significantly different changes. Although the number of subjects is too limited to confer a rigid statistical inference, the preset fNIRS study is the first to visualize the distinct neuropharmacological activation patterns between medication-naïve ADHD children with and without comorbid ASD.

\section{Limitations}

As discussed above, the current study has demonstrated that MPH-effect assessment in ADHD children using ANIRS can be applicable to medication-naïve ADHD children with and without ASD at elementary school ages. However, several issues need to be addressed before establishing its clinical utility.
First, in the current study, the diagnosis of ADHD with ASD was based on the DSM-5 criteria as in a former fMRI study using ICD10 [49]. However, the severity and characteristics of ASD comorbidity is better confirmed using a semi-structured assessment (e.g. Autism Diagnostic Observation Schedule, Module 4 [78] and Autism Diagnostic Interview-Revised [79]). For objective evaluation of ASD traits, semi-structured assessments are needed in the next study.

Second, the present study included a rather small sample size, and further research is needed to increase the sample size of ADHD children with ASD and strengthen the conclusions drawn. Nevertheless, the current study is meaningful in the sense that it reflects the realworld distribution of ADHD children with and without comorbid ASD [80,81].

Third, in the current study all of the patients were Japanese children. In order to generalize the different neuroactivation patterns of ADHD with ASD and that without ASD, and the differential neuropharmacological effects of $\mathrm{MPH}$ on the IFG/MFG, further racial-ethnic validation studies are needed.

\section{Conclusions}

To our knowledge, this is the first comparative fNIRS-based neuropharmacological study between pediatric ADHD with and without comorbid ASD. We demonstrated that MPHinduced neuroactivation patterns in ADHD children with and without ASD are associated with distinct disorder-specific abnormalities. ADHD subjects without ASD exhibited similar neuroactivation in the right IFG/MFG as typically developing children after $\mathrm{MPH}$ medication; conversely, ADHD children with ASD had different activation patterns. Therefore, these results suggest that ADHD with ASD is not simply a mixture of two pure disorders, but rather that it has its own distinct neuropathology.

As for clinical utility, in order to ensure successful early diagnosis and treatment of medication-naïve ADHD children without ASD, it is important to establish an objective biomarker to assess the effectiveness of MPH in initial interventions. The current method provides a promising possibility to enable discrimination between responders and non-responders at the earliest possible time. This should further render the effects of $\mathrm{MPH}$ treatment observable so as to motivate patient guardians to continue medication. 


\section{Acknowledgements}

We appreciate ELCS for English proofreading. We thank Illpop (http://illpop.com/animal_top01. htm) for kindly providing source pictures for experimental materials. We appreciate the members of the RISTEX group for their assistance. This work was supported in part by JST-RISTEX to $I D$, and the Grant-in-Aid for Scientific Research from the Japan Society for Promotion of Science (17H05959) to ID.

\section{References}

1. Diagnostic and statistical manual of mental disorders. (DSM-5). APA, Arlington (2013).

2. Dittmann RW, Wehmeier P M, Schacht A, et al. Atomoxetine treatment and ADHD-related difficulties as assessed by adolescent patients, their parents and physicians. Child. Adolesc. Psychiatry. Ment. Health 3(1), 21 (2009).

3. Pietrzak R H, Mollica C M, Maruff $P$, et al. Cognitive effects of immediate-release methylphenidate in children with attention-deficit/ hyperactivity disorder. Neurosci. Biobehav. Rev 30(8), 1225-1245 (2006).

4. Drechsler $R$, Brandeis $D$, Foldenyi $M$, et al. The course of neuropsychological functions in children with attention deficit hyperactivity disorder from late childhood to early adolescence. J. Child. Psycho.I Psychiatry 46(8), 824-836 (2005)

5. Classi P, Milton D, Ward S, et al. Social and emotional difficulties in children with ADHD and the impact on school attendance and healthcare utilization. Child. Adolesc. Psychiatry. Ment. Health 6(1), 33 (2012).

6. Power T J, Shapiro E S, DuPaul G J. Preparing psychologists to link systems of care in managing and preventing children's health problems. J. Pediatr. Psychol 28(2), 147-155 (2003).

7. Wolraich $M$, Brown L, Brown RT, et al. ADHD: clinical practice guideline for the diagnosis, evaluation, and treatment of attention-deficit/hyperactivity disorder in children and adolescents. Pediatrics 128(5), 1007-1022 (2011).

8. Hodgkins $P$, Shaw M, Coghill D, et al. Amfetamine and methylphenidate medications for attention-deficit/hyperactivity disorder: complementary treatment options. Eur. Child. Adolesc. Psychiatry 21(9), 477-492 (2012).

9. Spencer $T J, A D H D$ treatment across the life cycle. J. Clin. Psychiatry 65 Suppl 3, 22-26 (2004).

10. Rubia K, Halari R, Mohammad A M, et al. Methylphenidate normalizes frontocingulate underactivation during error processing in attention-deficit/hyperactivity disorder. Biol. Psychiatry 70(3), 255-262 (2011).

11. Wilens $T E$, Effects of methylphenidate on the catecholaminergic system in attention-deficit/hyperactivity disorder. J. Clin. Psychopharmacol 28(3 Suppl 2), S46-53 (2008).

12. Arnsten A F. Stimulants: Therapeutic actions in ADHD. Neuropsychopharmacology 31(11), 2376-2383 (2006).

13. Taurines $R$, Schwenck $C$, Westerwald $E$, et al. ADHD and autism: differential diagnosis or overlapping traits? A selective review. Atten. Defic. Hyperact. Disord 4(3), 115-139 (2012).

14. Leitner $Y$. The co-occurrence of autism and attention deficit hyperactivity disorder in children - what do we know? Front. Hum. Neurosci 8(1), 268 (2014).

15. Newcorn J H, Kratochvil C J, Allen A J, et al. Atomoxetine and osmotically released methylphenidate for the treatment of attention deficit hyperactivity disorder: acute comparison and differential response. Am. J.Psychiatry 165(6), 721-730 (2008).

16. Zhu C Z, Zang Y F, Cao Q J, et al. Fisher discriminative analysis of resting-state brain function for attention-deficit/hyperactivity disorder. Neurolmage 40(1), 110-120 (2008).

17. Barkley R A, Issues in the diagnosis of attention-deficit/hyperactivity disorder in children. Brain. Dev 25(2), 77-83 (2003).

18. Okamoto M, Dan H, Shimizu K, et al. Multimodal assessment of cortical activation during apple peeling by NIRS and fMRI. Neurolmage 21(4), 1275-1288 (2004).

19. Herrmann M J, Ehlis A C, and Fallgatter A J. Bilaterally reduced frontal activation during a verbal fluency task in depressed patients as measured by near-infrared spectroscopy. J.Neuropsychiatry. Clin. Neurosci 16(2), 170-175 (2004).

20. Hock C, Villringer K, Müller-Spahn F, et al. Decrease in parietal cerebral hemoglobin oxygenation during performance of a verbal fluency task in patients with Alzheimer's disease monitored by means of near-infrared spectroscopy (NIRS) - correlation with simultaneous rCBF-PET measurements. Brain. Research 755(2), 293-303 (1997).

21. Matsuo K, Kato T, Fukuda M, et al. Alteration of hemoglobin oxygenation in the frontal region in elderly depressed patients as measured by near-infrared spectroscopy. $J$ Neuropsychiatry Clin Neurosci. 12(4), 465-471 (2000).

22. Matsuo K, Taneichi K, Matsumoto A, et al. Hypoactivation of the prefrontal cortex during verbal fluency test in PTSD: a near-infrared spectroscopy study. Psychiatry. Res 124(1), 1-10 (2003).

23. Shinba T, Nagano M, Kariya N, et al. Near-in- frared spectroscopy analysis of frontal lobe dysfunction in schizophrenia. Biol. Psychiatry. 55(2), 154-164 (2004).

24. Suto T, Fukuda $M$, Ito $M$, et al. Multichannel near-infrared spectroscopy in depression and schizophrenia: cognitive brain activation study. Biol. Psychiatry. 55(5), 501-511 (2004).

25. Herrmann M J, Plichta M M, Ehlis A C, et al. Optical topography during a Go-NoGo task assessed with multi-channel near-infrared spectroscopy. Behav. Brain. Res.= 160(1), 135140 (2005).

26. Okamoto M, Matsunami M, Dan H, et al. Prefrontal activity during taste encoding: an fNIRS study. Neurolmage. $=31(2), 796-806$ (2006).

27. Moriai-Izawa A, Dan H, Dan I, et al. Multichannel fNIRS assessment of overt and covert confrontation naming. Brain . Lang. 121(3), 185-193 (2012).

28. Strangman G, Boas D A, Sutton JP. Non-invasive neuroimaging using near-infrared light. Biol. Psychiatry 52(7), 679-693 (2002).

29. Rubia K, Halari R, Cubillo A, et al. Methylphenidate normalizes fronto-striatal underactivation during interference inhibition in medication-naive boys with attention-deficit hyperactivity disorder. Neuropsychopharmacology 36(8), 1575 (2011).

30. Rubia K, Halari R, Mohammad A-M, et al. Methylphenidate normalizes frontocingulate underactivation during error processing in attention-deficit/hyperactivity disorder. Biological. psychiatry 70(3), 255-262 (2011).

31. Rubia K, Halari R, Cubillo A, et al. Methylphenidate normalises activation and functional connectivity deficits in attention and motivation networks in medication-naive children with ADHD during a rewarded continuous performance task. Neuropharmacology 57 (78), 640-652 (2009).

32. Durston $\mathrm{S}$, Tottenham N T, Thomas K M, et al. Differential patterns of striatal activation in young children with and without ADHD. Biol. Psychiatry.= 53(10), 871-878 (2003).

33. Ishii-Takahashi A, Takizawa R, Nishimura Y, et al. Neuroimaging-aided prediction of the effect of methylphenidate in children with attention-deficit hyperactivity disorder: a randomized controlled trial. Neuropsychopharmacology 40(12), 2676 (2015).

34. Araki A, Ikegami M, Okayama A, et al. Improved prefrontal activity in AD/HD children 
treated with atomoxetine: A NIRS study. Brain. And. Development 37(1), 76-87 (2015).

35. Nakanishi Y, Ota T, lida J, et al. Differential therapeutic effects of atomoxetine and methylphenidate in childhood attention deficit/hyperactivity disorder as measured by near-infrared spectroscopy. Child. And. Adolescent. Psychiatry. And. Mental. Health 11(1), 26 (2017).

36. Nagashima M, Monden $Y$, Dan I, et al. Neuropharmacological effect of methylphenidate on attention network in children with attention deficit hyperactivity disorder during oddball paradigms as assessed using functional near-infrared spectroscopy. Neurophotonics 1(1), 015001 (2014).

37. Nagashima M, Monden Y, Dan I, et al. Neuropharmacological effect of atomoxetine on attention network in children with attention deficit hyperactivity disorder during oddball paradigms as assessed using functional near-infrared spectroscopy. Neurophotonics 1(2), 025007 (2014).

38. Nagashima M, Monden Y, Dan I, et al. Acute neuropharmacological effects of atomoxetine on inhibitory control in ADHD children: a fNIRS study. Neurolmage. Clin. 6(1), 192201 (2014).

39. Matsuura N, Ishitobi M, Arai S, et al. Effects of methylphenidate in children with attention deficit hyperactivity disorder: a near-infrared spectroscopy study with CANTAB $^{\circledast}$. Child. And. Adolescent. Psychiatry. And. Mental. health 8(1), 273 (2014).

40. Monden Y, Dan H, Nagashima M, et al. Right prefrontal activation as a neuro-functional biomarker for monitoring acute effects of methylphenidate in ADHD children: An fNIRS study. Neurolmage. Clin. 1(1), 131-140 (2012).

41. Schecklmann M, Schaldecker M, Aucktor $S$, et al. Effects of methylphenidate on olfaction and frontal and temporal brain oxygenation in children with ADHD. Journal. of. psychiatric. research 45(11), 1463-1470 (2011).

42. Sanefuji $M$, Yamashita $H$, Torisu $H$, et al. Altered strategy in short-term memory for pictures in children with attention-deficit/ hyperactivity disorder: a near-infrared spectroscopy study. Psychiatry. Res 223(1), 37-42 (2014)

43. Monden $Y$, Dan $H$, Nagashima $M$, et al. Clinically-oriented monitoring of acute effects of methylphenidate on cerebral hemodynamics in ADHD children using fNIRS. Clin. Neurophysiol 123(6), 1147-1157 (2012).

44. Monden Y, Dan I, Nagashima M, et al. Individual classification of ADHD children by right prefrontal hemodynamic responses during a go/no-go task as assessed by fNIRS. Neurolmage. Clin 9(1), 1-12 (2015).

45. Craig F, Lamanna A L, Margari F, et al. Over- lap Between Autism Spectrum Disorders and Attention Deficit Hyperactivity Disorder: Searching for Distinctive/Common Clinical Features. Autism. Res 8(3), 328-337 (2015).

46. Craig F, Margari F, Legrottaglie A R, et al. A review of executive function deficits in autism spectrum disorder and attention-deficit/hyperactivity disorder. Neuropsychiatr. Dis. Treat 12, 1191-1202 (2016).

47. Arnold L E, Aman M G, Li X, et al. Research Units of Pediatric Psychopharmacology (RUPP) autism network randomized clinical trial of parent training and medication: oneyear follow-up. J. Am. Acad. Child. Adolesc. Psychiatry 51(11), 1173-1184 (2012).

48. Tye C, Asherson P, Ashwood K L, et al. Attention and inhibition in children with ASD, ADHD and co-morbid ASD + ADHD: an event-related potential study. Psychol. Med 44(5), 1101-1116 (2014).

49. Chantiluke K, Christakou A, Murphy C M, et al. Disorder-specific functional abnormalities during temporal discounting in youth with Attention Deficit Hyperactivity Disorder (ADHD), Autism and comorbid ADHD and Autism. Psychiatry. Res 223(2), 113-120 (2014).

50. Moll G H, Hause S, Ruther E, et al. Early methylphenidate administration to young rats causes a persistent reduction in the density of striatal dopamine transporters. J. Child. Adolesc. Psychopharmacol 11(1), 15-24 (2001).

51. Nakao T, Radua J, Rubia K, et al. Gray matter volume abnormalities in ADHD: voxel-based meta-analysis exploring the effects of age and stimulant medication. Am. J. Psychiatry 168(11), 1154-1163 (2011).

52. Bledsoe J, Semrud-Clikeman M, Pliszka SR. A magnetic resonance imaging study of the cerebellar vermis in chronically treated and treatment-naive children with attention-deficit/hyperactivity disorder combined type. Biol. Psychiatry 65(7), 620624 (2009).

53. Shaw P, Sharp W S, Morrison M, et al. Psychostimulant treatment and the developing cortex in attention deficit hyperactivity disorder. Am. J. Psychiatry 166(1), 58-63 (2009).

54. Yamazaki K, ADHD-RS-IV Japanese version, in Japanese guideline for the diagnosis and treatment of attention deficit hyperactivity disorder (ADHD). Jiho, Tokyo (2013).

55. DuPaul G J, Anastopoulos AD, Power T J, et al. Parent ratings of attention-deficit/ hyperactivity disorder symptoms: Factor structure and normative data. Journal. of. Psychopathology. and Behavioral. Assessment 20(1), 83-102 (1998).

56. Vaidya C J, Austin G, Kirkorian G, et al. Selective effects of methylphenidate in attention deficit hyperactivity disorder: a functional magnetic resonance study. Proc. Natl .Acad. Sci U S A 95(24), 14494-14499 (1998).

57. Liddle P F, Kiehl K A, Smith A M. Event-related $\mathrm{fMRI}$ study of response inhibition. Hum. Brain. Mapp 12(2), 100-109 (2001).

58. Menon V, Adleman N E, White C D, et al. Error-related brain activation during a Go/ NoGo response inhibition task. Hum Brain. Mapp 12(3), 131-143 (2001).

59. Dillo W, Goke A, Prox-Vagedes V, et al. Neuronal correlates of ADHD in adults with evidence for compensation strategies--a functional MRI study with a Go/No-Go paradigm. Ger. Med. Sci. 8, Doc09 (2010).

60. Cope M, Delpy D T, Reynolds E O, et al. Methods of quantitating cerebral near infrared spectroscopy data. Advances. In. Experimental. Medicine. And. Biology 222(1), 183-189 (1988).

61. Maki A, Yamashita Y, Ito Y, et al. Spatial and temporal analysis of human motor activity using noninvasive NIR topography. Medical. Physics 22(12), 1997-2005 (1995).

62. Garavan H, Ross T J, Stein E A, Right hemispheric dominance of inhibitory control: an event-related functional MRI study. Proc. Natl. Acad. Sci. U S A 96(14), 8301-8306 (1999).

63. Tsuzuki D, Cai D S, Dan H, et al. Stable and convenient spatial registration of standalone NIRS data through anchor-based probabilistic registration. Neurosci. Res 72(2), 163-171 (2012).

64. Tsuzuki D, Dan I, Spatial registration for functional near-infrared spectroscopy: from channel position on the scalp to cortical location in individual and group analyses. Neurolmage 85 Pt 1, 92-103 (2014).

65. Tsuzuki D, Jurcak V, Singh A K, et al. Virtual spatial registration of stand-alone fNIRS data to MNI space. Neurolmage 34(4), 15061518 (2007).

66. Shattuck D W, Mirza M, Adisetiyo V, et al. Construction of a 3D probabilistic atlas of human cortical structures. Neurolmage 39(3), 1064-1080 (2008).

67. Rorden $C$ and Brett M, Stereotaxic display of brain lesions. Behav. Neurol 12(4), 191-200 (2000).

68. Khan A, Khan S, Brown W A, Are placebo controls necessary to test new antidepressants and anxiolytics? Int. J. Neuropsychopharmacol 5(3), 193-197 (2002).

69. Khan A, Warner H A, Brown W A, Symptom reduction and suicide risk in patients treated with placebo in antidepressant clinical trials: an analysis of the Food and Drug Administration database. Arch. Gen. Psychiatry 57(4), 311-317 (2000).

70. Rutherford B R, Roose S P, A model of pla- 


\section{A Functional Near-Infrared Spectroscopy Study}

cebo response in antidepressant clinical trials. Am. J. Psychiatry 170(7), 723-733 (2013)

71. Sinyor $\mathrm{M}$, Levitt $\mathrm{A} J$, Cheung $\mathrm{A} \mathrm{H}$, et al. Does inclusion of a placebo arm influence response to active antidepressant treatment in randomized controlled trials? Results from pooled and meta-analyses. J. Clin. Psychiatry 71(3), 270-279 (2010).

72. Asahi S, Okamoto Y, Okada G, et al. Negative correlation between right prefrontal activity during response inhibition and impulsiveness: a fMRI study. Eur. Arch. Psychiatry. Clin. Neurosci 254(4), 245-251 (2004).

73. Tamm L, Menon V, Ringel J, et al. Event-related FMRI evidence of frontotemporal involvement in aberrant response inhibition and task switching in attention-deficit/hyperactivity disorder. J. Am. Acad. Child. Adolesc. Psychiatry 43(11), 1430-1440 (2004).
74. Chikazoe J. Localizing performance of go/ no-go tasks to prefrontal cortical subregions. Curr. Opin. Psychiatry 23(3), 267-272 (2010).

75. Niendam T A, Laird A R, Ray K L, et al. Meta-analytic evidence for a superordinate cognitive control network subserving diverse executive functions. Cogn. Affect. Behav. Neurosci 12(2), 241-268 (2012).

76. Buchsbaum BR, Greer S, Chang WL, et al. Meta-analysis of neuroimaging studies of the Wisconsin card-sorting task and component processes. Hum. Brain. Mapp 25(1), 35-45 (2005).

77. Simmonds DJ, Pekar JJ, Mostofsky SH. Meta-analysis of Go/No-go tasks demonstrating that $\mathrm{FMRI}$ activation associated with response inhibition is task-dependent. Neuropsychologia 46(1), 224-232 (2008).

78. Lord C, Risi S, Lambrecht L, et al. The autism diagnostic observation schedule-generic: a standard measure of social and communication deficits associated with the spectrum of autism. J. Autism. Dev. Disord 30(3), 205-223 (2000).

79. Lord C, Rutter M, and Le Couteur A, Autism Diagnostic Interview-Revised: a revised version of a diagnostic interview for caregivers of individuals with possible pervasive developmental disorders. J. Autism. Dev. Disord 24(5), 659-685 (1994).

80. Rommelse NN, Franke B, Geurts HM, et al. Shared heritability of attention-deficit/ hyperactivity disorder and autism spectrum disorder. Eur. Child. Adolesc. Psychiatry 19(3), 281-295 (2010).

81. Jang J, Matson JL, Williams LW, et al. Rates of comorbid symptoms in children with ASD, ADHD, and comorbid ASD and ADHD. Res. Dev. Disabil 34(8), 2369-2378 (2013). 